\title{
General Solution and Observability of Singular Differential Systems with Delay
}

\author{
Jiang Wei \\ School of Mathematical Science, University of Anhui, Hefei, Anhui 230039, China \\ Correspondence should be addressed to Jiang Wei; jiangwei89018@126.com
}

Received 25 July 2013; Accepted 4 October 2013

Academic Editor: Shengqiang Liu

Copyright (C) 2013 Jiang Wei. This is an open access article distributed under the Creative Commons Attribution License, which permits unrestricted use, distribution, and reproduction in any medium, provided the original work is properly cited.

We study singular differential systems with delay. A general description for the solutions of singular differential systems with delay is given and a necessary and sufficient condition for exact observability of singular differential systems with delay is derived.

\section{Introduction}

In many practical systems, such as economic systems, power systems, biological systems, ecosystems, and so on, due to the transmission of information need time span, usually there are the phenomena of delay. Up to now, many authors have paid much attention to the study for delay, and many excellent results have been given [1-5]. We notice that in some systems we must consider their character of dynamic state and static state synchronously, these result in many good consequences for singular systems that have been obtained recently [6-9].

We must emphasize that there are a lot of systems in which there exist the phenomena of delay and singular simultaneously, we call such systems as the singular differential systems with delay. These systems have many special characters. If we want to describe them more exactly, more accurately design them, and more effectively control them, we must pay tremendous endeavor to investigate them, but that is obviously very difficult. In [3, 10-19], authors have discussed such systems, and got some consequences. But in the study for such systems, there are still many problems to be considered.

Generally, the singular differential control systems with delay can be written as

$$
\begin{gathered}
E \dot{x}(t)=F(x(t), x(t-\tau), u(t), t), \quad t \geq 0, \\
x(t)=\varphi(t), \quad-\tau \leq t \leq 0,
\end{gathered}
$$

where $x(t) \in R^{n}$ is a state vector, $u(t) \in R^{l}$ is a control vector, $E \in R^{n \times n}$ is a singular matrix, $\varphi \in C=C\left([-\tau, 0], R^{n}\right)$ is an admissible initial state functional, $C=C\left([-\tau, 0], R^{n}\right)$ is the Banach space of continuous functions mapping the interval $[-\tau, 0]$ into $R^{n}$ with the topology of uniform convergence, the norm of an element $\varphi$ in $C$ can be designated as $|\varphi|=$ $\sup _{\theta \in[-\tau, 0]}|\varphi(\theta)|$, and $F \in C^{1}\left(R^{n} \times R^{n} \times R^{l} \times R, R^{n}\right)$.

The autonomous linear form of (1) can be written as

$$
\begin{gathered}
E \dot{x}(t)=A x(t)+B x(t-\tau)+D u(t)+f(t), \quad t \geq 0, \\
x(t)=\varphi(t), \quad-\tau \leq t \leq 0,
\end{gathered}
$$

where $x(t) \in R^{n}$ is a state vector $[6,7], u(t) \in R^{l}$ is a control vector [6,7], $E \in R^{n \times n}$ is a singular matrix, $A, B \in R^{n \times n}$, and $D \in R^{n \times l}$ are constant matrix, $f(t) \in R^{n}$ is a sufficiently smooth function, and $\varphi(t)$ is the initial state function.

Usually, the output functions of (2) can be written as:

$$
y(t)=C x(t)+H u(t),
$$

where $y(t) \in R^{m}$ is an output vector [6, 7], $x(t) \in R^{n}$ is a state vector, $u(t) \in R^{l}$ is a control vector, and $C \in R^{m \times n}$ and $H \in R^{m \times l}$ are constant matrices.

Definition 1. Singular differential control systems with delay (2) and (3) are observable on $\left[t_{1}, t_{2}\right]$ if the initial function $\varphi(t)$ in $[-\tau, 0]$ can be uniquely determined from the knowledge of the control $u(\cdot)$ and observation $y(\cdot)$ over $\left[t_{1}, t_{2}\right]$.

Remark 2. The observability concept in Definition 1 comes from [4], which is sometimes called exact observability. 
Recently, many authors have discussed the observability of singular differential control systems without delay [6-8], but for such systems the discussion is much easier than that for singular differential control systems with delay. This is because singular differential control systems without delay can be changed as two autocephalous systems $[7,8]$ as follows:

$$
\begin{gathered}
\dot{z}_{1}(t)=A_{1} z_{1}(t)+G_{1} u(t)+f_{1}(t), \\
N \dot{z}_{2}(t)=z_{2}(t)+B u(t)+f_{2}(t),
\end{gathered}
$$

and they can be discussed, respectively. But for singular differential systems with delay (2), the canonical forms are

$$
\begin{gathered}
\dot{x}_{1}(t)=A_{1} x_{1}(t)+B_{11} x_{1}(t-\tau)+B_{12} x_{2}(t-\tau) \\
+C_{1} u(t)+f_{1}(t), \quad t \geq 0 \\
N \dot{x}_{2}(t)=x_{2}(t)+B_{21} x_{1}(t-\tau)+B_{22} x_{2}(t-\tau) \\
\quad+C_{2} u(t)+f_{2}(t), \quad t \geq 0 \\
x_{1}(t)=\varphi_{1}(t), \quad-\tau \leq t \leq 0, \\
x_{2}(t)=\varphi_{2}(t), \quad-\tau \leq t \leq 0
\end{gathered}
$$

which generally cannot be discussed, respectively.

In papers $[10,16]$, we have had some results about the controllability of singular control systems with control-delay and the controllability of singular control systems with statedelay, respectively. As the corresponding concept of controllability, observability is obviously and important concept. As we know, up to now, there exists hardly any discussion on the observability of singular differential systems with delay.

In this paper, we study singular differential systems with delay. Firstly, for the singular differential systems with delay, the general solutions will be given. Then, for the observability of singular differential systems with delay, we will give criterion.

\section{General Solutions of Singular Differential Systems with Delay}

In the study of the observability of singular differential systems with delay, we will use some concepts and conclusions. Now, we give some of them, and specially give the general solutions of singular differential systems with delay (2).

Definition 3. Let $E$ be a square matrix, if there exists a matrix $E^{d}$ satisfy $1^{0} \quad E E^{d}=E^{d} E, 2^{0} \quad E^{d} E E^{d}=E^{d}, 3^{0} \quad\left(I-E^{d} E\right)$ $E^{l}=0$, we call $E^{d}$ the Drazin inverse matrix of matrix $E$, simply $D$-inverse matrix. Where $l$ is the index of matrix $E$; it is the smallest nonnegative integer which makes $\operatorname{rank}\left(E^{l+1}\right)=$ $\operatorname{rank}\left(E^{l}\right)$ be true.

From [3] or [7], we have the following.

Lemma 4. For any square matrix $E$, its Drazin inverse matrix $E^{d}$ is existent and unique. If the Jordan normalized form of $E$ is $E=T\left(\begin{array}{cc}J_{1} & 0 \\ 0 & J_{0}\end{array}\right) T^{-1}$, then $E^{d}=T\left(\begin{array}{cc}J_{1}^{-1} & 0 \\ 0 & 0\end{array}\right) T^{-1}$. Here, $J_{0}$ is a nilpotent matrix, $J_{1}$ and $T$ are invertible matrices.
Consider the following systems

$$
\begin{gathered}
E \dot{x}(t)=A x(t)+B x(t-\tau), \quad t \geq 0, \\
x(t)=\varphi(t), \quad-\tau \leq t \leq 0,
\end{gathered}
$$

and systems:

$$
\begin{gathered}
E \dot{x}(t)=A x(t)+B x(t-\tau)+D u(t)+f(t), \quad t \geq 0, \\
x(t) \equiv 0, \quad-\tau \leq t \leq 0 .
\end{gathered}
$$

It is not difficult to prove the following.

Lemma 5. If $\bar{x}(t)$ and $\widehat{x}(t)$ are, respectively, the solution to (6) and (7), then $x(t)=\bar{x}(t)+\widehat{x}(t)$ is the solution to (2).

Definition 6. Let $X(t) \in R^{n \times n}, X(t)$ is called as the first class foundation solution of singular differential systems with delay, if it satisfies matrix equations the following:

$$
\begin{gathered}
E \dot{X}(t)=A X(t)+B X(t-\tau), \quad t \geq 0, \\
X(t)= \begin{cases}E E^{d}, & t=0, \\
0, & -\tau \leq t<0 .\end{cases}
\end{gathered}
$$

Definition 7. Let $Y(t) \in R^{n \times n}, Y(t)$ is called as the second class foundation solution of singular differential systems with delay, if it satisfies matrix equations the following:

$$
\begin{gathered}
E \dot{Y}(t)=A Y(t)+B Y(t-\tau)+\left(I-E E^{d}\right) \delta(t), \quad t \geq 0, \\
Y(t)= \begin{cases}I-E E^{d}, & t=0, \\
0, & -\tau \leq t<0,\end{cases}
\end{gathered}
$$

where $\delta(t)$ is a delta function or impulse function (see [3] or [7]).

Lemma 8. For delta function $\delta(t)$, one has

$$
\int_{0}^{t} \delta(t-s) f(s) d s=f(t) .
$$

This Lemma is well known. The proof will be omitted.

Lemma 9. For any square matrix E, one has:

$$
(E+I)\left(I-E E^{d}\right)\left(I+E\left(I-E E^{d}\right)\right)^{-1}=I-E E^{d} .
$$

Proof. Let $I$ be an identity matrix with appropriate dimension, and

$$
E=T\left(\begin{array}{cc}
J_{1} & 0 \\
0 & J_{0}
\end{array}\right) T^{-1},
$$

from Lemma 4, we have

$$
E^{d}=T\left(\begin{array}{cc}
J_{1}^{-1} & 0 \\
0 & 0
\end{array}\right) T^{-1} .
$$


For $J_{0}$ is a nilpotent matrix, $J_{0}+I$ is invertible. Consider the following

$$
\begin{aligned}
& I-E E^{d}=T\left(\begin{array}{ll}
I & 0 \\
0 & I
\end{array}\right) T^{-1} \\
& -T\left(\begin{array}{cc}
J_{1} & 0 \\
0 & J_{0}
\end{array}\right) T^{-1} T\left(\begin{array}{cc}
J_{1}^{-1} & 0 \\
0 & 0
\end{array}\right) T^{-1} \\
& =T\left(\begin{array}{ll}
I & 0 \\
0 & I
\end{array}\right) T^{-1}-T\left(\begin{array}{ll}
I & 0 \\
0 & 0
\end{array}\right) T^{-1} \\
& =T\left(\begin{array}{ll}
0 & 0 \\
0 & I
\end{array}\right) T^{-1} \\
& (E+I)\left(I-E E^{d}\right)=T\left(\begin{array}{cc}
J_{1}+I & 0 \\
0 & J_{0}+I
\end{array}\right) T^{-1} T\left(\begin{array}{ll}
0 & 0 \\
0 & I
\end{array}\right) T^{-1} \\
& =T\left(\begin{array}{cc}
0 & 0 \\
0 & J_{0}+I
\end{array}\right) T^{-1} \\
& I+E\left(I-E E^{d}\right)=T\left(\begin{array}{ll}
I & 0 \\
0 & I
\end{array}\right) T^{-1} \\
& +T\left(\begin{array}{cc}
J_{1} & 0 \\
0 & J_{0}
\end{array}\right) T^{-1} T\left(\begin{array}{ll}
0 & 0 \\
0 & I
\end{array}\right) T^{-1} \\
& =T\left(\begin{array}{cc}
I & 0 \\
0 & J_{0}+I
\end{array}\right) T^{-1} \\
& I-E E^{d}=T\left(\begin{array}{ll}
0 & 0 \\
0 & I
\end{array}\right) T^{-1} \\
& =T\left(\begin{array}{cc}
0 & 0 \\
0 & J_{0}+I
\end{array}\right) T^{-1} T\left(\begin{array}{cc}
I & 0 \\
0 & J_{0}+I
\end{array}\right)^{-1} T^{-1} \\
& =(E+I)\left(I-E E^{d}\right)\left(I+E\left(I-E E^{d}\right)\right)^{-1} .
\end{aligned}
$$

That means (11) is true.

Definition 10. If $\operatorname{det}(\lambda E-A) \not \equiv 0$, we call matrix couple $(E, A)$ regular. If $(A, E)$ is regular, we call system (2) regular.

Remark 11. Using the method of [3], we can prove that if $(E, A)$ is regular, systems (2) (for any consistent initial state function $\varphi(t)),(8)$, and (9) are solvable.

Theorem 12. Suppose that matrix couple $(A, E)$ is regular, $\bar{x}(t)$ is the solution of (6); then when $t \geq \tau$,

$$
\begin{array}{r}
\bar{x}(t)=\left[X(t)+Y(t)\left(I+E\left(I-E E^{d}\right)\right)^{-1} E\right] \varphi(0) \\
+\int_{-\tau}^{0}\left[X(t-\theta-\tau) E^{d} B+Y(t-\theta-\tau)\right. \\
\left.\times\left(I+E\left(I-E E^{d}\right)\right)^{-1} B\right] \varphi(\theta) d \theta,
\end{array}
$$

when $0 \leq t \leq \tau$

$$
\begin{array}{r}
\bar{x}(t)=\left[X(t)+Y(t)\left(I+E\left(I-E E^{d}\right)\right)^{-1} E\right] \varphi(0) \\
+\int_{-\tau}^{t-\tau}\left[X(t-\theta-\tau) E^{d} B+Y(t-\theta-\tau)\right. \\
\left.\times\left(I+E\left(I-E E^{d}\right)\right)^{-1} B\right] \varphi(\theta) d \theta
\end{array}
$$

where $X(t)$ is the first class of foundation solution of singular differential systems with delay, $Y(t)$ is the second class of foundation solution of singular differential systems with delay.

To prove Theorem 12, we can partition homogeneous singular differential systems with delay (6) into two classes of systems as follows:

$$
\begin{gathered}
E \dot{x}(t)=A x(t)+B x(t-\tau), \quad t \geq \tau, \\
E \dot{x}(t)=A x(t)+E E^{d} B \varphi(t-\tau), \quad 0 \leq t \leq \tau, \\
x(t)=E E^{d} \varphi(t), \quad-\tau \leq t \leq 0, \\
E \dot{x}(t)=A x(t)+B x(t-\tau), \quad t \geq \tau, \\
E \dot{x}(t)=A x(t)+\left(I-E E^{d}\right) B \varphi(t-\tau), \quad 0 \leq t \leq \tau, \\
x(t)=\left(I-E E^{d}\right) \varphi(t), \quad-\tau \leq t \leq 0 .
\end{gathered}
$$

We can easily prove the following.

Lemma 13. If $x_{1}(t)$, and $x_{2}(t)$ are respectively the solution of (17) and (18), then $\bar{x}(t)=x_{1}(t)+x_{2}(t)$ is the solution of (6).

Lemma 14. Suppose that matrix couple $(A, E)$ is regular, then the solution of (17) can be written as

$$
x_{1}(t)=\left\{\begin{array}{l}
X(t) \varphi(0) \\
\quad+\int_{-\tau}^{0} X(t-\theta-\tau) E^{d} B \varphi(\theta) d \theta, \quad t \geq \tau, \\
X(t) \varphi(0) \\
\quad+\int_{0}^{t} X(t-\theta) E^{d} B \varphi(\theta-\tau) d \theta, \quad 0 \leq t \leq \tau,
\end{array}\right.
$$

where $X(t)$ is the first class of foundation solution of singular differential systems with delay. 
Proof. Since $X(t)$ is the first class of foundation solution of singular differential systems with delay, $X(t)$ satisfies matrix equations (8). Take Laplace transform for (8), we have

$$
\begin{aligned}
& \int_{0}^{\infty} e^{-\lambda t}(E \dot{X}(t)) d t \\
&= E \int_{0}^{\infty} e^{-\lambda t} \dot{X}(t) d t \\
&=E \lambda \int_{0}^{\infty} e^{-\lambda t} X(t) d t-E X(0) \\
&= \lambda E L(X(t))-E E E^{d}, \\
& \int_{0}^{\infty} e^{-\lambda t}(A X(t)+B X(t-\tau)) d t \\
&=A \int_{0}^{\infty} e^{-\lambda t} X(t) d t \\
& \quad+B \int_{0}^{\infty} e^{-\lambda t} X(t-\tau) d t \\
&=A L(X(t))+B \int_{-\tau}^{\infty} e^{-\lambda t-\lambda \tau} X(t) d t \\
&= A L(X(t))+B e^{-\lambda \tau} \int_{0}^{\infty} e^{-\lambda t} X(t) d t \\
&\left.A e^{-\lambda \tau}\right) L(X(t)) .
\end{aligned}
$$

That is,

$$
\lambda E L(X(t))-E E E^{d}=\left(A+B e^{-\lambda \tau}\right) L(X(t)) .
$$

Because $(E, A)$ is regular, for $\operatorname{real}(\lambda)$ large enough, $\lambda E-$ $A-B e^{-\lambda \tau}$ is invertible, so we have

$$
L(X(t))=\left(\lambda E-A-B e^{-\lambda \tau}\right)^{-1} E E E^{d} .
$$

If $x_{1}(t)$ is the solution of (17), we have

$$
\begin{aligned}
\int_{0}^{\infty} e^{-\lambda t}\left(E \dot{x}_{1}(t)\right) d t= & E \int_{0}^{\infty} e^{-\lambda t} \dot{x}_{1}(t) d t \\
= & E \lambda \int_{0}^{\infty} e^{-\lambda t} x_{1}(t) d t-E x_{1}(0) \\
= & \lambda E L\left(x_{1}(t)\right)-E E E^{d} \varphi(0), \\
\int_{0}^{\infty} e^{-\lambda t}\left(E \dot{x}_{1}(t)\right) d t= & \int_{0}^{\tau} e^{-\lambda t}\left(E \dot{x}_{1}(t)\right) d t \\
& +\int_{\tau}^{\infty} e^{-\lambda t}\left(E \dot{x}_{1}(t)\right) d t \\
= & A \int_{0}^{\tau} e^{-\lambda t} x_{1}(t) d t \\
& +\int_{0}^{\tau} E E^{d} B e^{-\lambda t} \varphi(t-\tau) d t \\
& +A \int_{\tau}^{\infty} e^{-\lambda t} x_{1}(t) d t
\end{aligned}
$$

$$
\begin{aligned}
& +B \int_{\tau}^{\infty} e^{-\lambda t} x_{1}(t-\tau) d t \\
= & A \int_{0}^{\infty} e^{-\lambda t} x_{1}(t) d t \\
& +B e^{-\lambda \tau} \int_{0}^{\infty} e^{-\lambda t} x_{1}(t) d t \\
& +\int_{0}^{\tau} E E^{d} B e^{-\lambda t} \varphi(t-\tau) d t \\
= & A L\left(x_{1}(t)\right)+B e^{-\lambda \tau} L\left(x_{1}(t)\right) \\
& +\int_{0}^{\tau} E E^{d} B e^{-\lambda t} \varphi(t-\tau) d t .
\end{aligned}
$$

That is,

$$
\begin{aligned}
\left(\lambda E-A-B e^{-\lambda \tau}\right) L\left(x_{1}(t)\right) & \\
& =E E E^{d} \varphi(0)+\int_{0}^{\tau} E E^{d} B e^{-\lambda t} \varphi(t-\tau) d t .
\end{aligned}
$$

Since $E^{d}=E E^{d} E^{d}$, from (22), we can see that

$$
\begin{aligned}
L\left(x_{1}(t)\right)= & \left(\lambda E-A-B e^{-\lambda \tau}\right)^{-1} E E E^{d} \varphi(0) \\
& +\int_{0}^{\tau}\left(\lambda E-A-B e^{-\lambda \tau}\right)^{-1} \\
& \times E E E^{d} E^{d} B e^{-\lambda t} \varphi(t-\tau) d t \\
= & L(X(t)) \varphi(0) \\
& +\int_{0}^{\tau} L(X(t)) E^{d} B e^{-\lambda s} \varphi(s-\tau) d s .
\end{aligned}
$$

Define an auxiliary function $\omega:[-\tau, \infty) \rightarrow[0,1]$ as follows:

$$
\omega(t)= \begin{cases}0, & t \geq 0 \\ 1, & t<0\end{cases}
$$

we have

$$
\begin{aligned}
\int_{0}^{\tau} L & (X(t)) E^{d} B e^{-\lambda s} \varphi(s-\tau) d s \\
& =L(X(t)) \int_{0}^{\tau} e^{-\lambda t} E^{d} B \varphi(t-\tau) d t \\
& =L(X(t)) \int_{0}^{\infty} e^{-\lambda t} E^{d} B \varphi(t-\tau) \omega(t-\tau) d t \\
& =L(X(t)) L\left(E^{d} B \varphi(t-\tau) \omega(t-\tau)\right) \\
& =L\left(\int_{0}^{t} X(t-\theta) E^{d} B \varphi(\theta-\tau) \omega(\theta-\tau) d \theta\right)
\end{aligned}
$$

Then, we have

$$
\begin{aligned}
x_{1}(t)= & X(t) \varphi(0) \\
& +\int_{0}^{t} X(t-\theta) E^{d} B \varphi(\theta-\tau) \omega(\theta-\tau) d \theta .
\end{aligned}
$$


When $t \geq \tau$,

$$
\begin{aligned}
x_{1}(t)= & X(t) \varphi(0) \\
& +\int_{-\tau}^{0} X(t-\theta-\tau) E^{d} B \varphi(\theta) d \theta,
\end{aligned}
$$

when $0 \leq t \leq \tau$,

$$
\begin{aligned}
x_{1}(t)= & X(t) \varphi(0) \\
& +\int_{0}^{t} X(t-\theta) E^{d} B \varphi(\theta-\tau) d \theta .
\end{aligned}
$$

The proof of Theorem 12 is completed.

Lemma 15. Suppose that matrix couple $(A, E)$ is regular, then the solution of (18) can be written as

$x_{2}(t)$

$$
=\left\{\begin{array}{cc}
Y(t)\left(I+E\left(I-E E^{d}\right)\right)^{-1} \varphi(0) & \\
+\int_{-\tau}^{0} Y(t-\theta-\tau)\left(I+E\left(I-E E^{d}\right)\right)^{-1} & \\
\quad \times B \varphi(\theta) d \theta, & \\
Y(t)\left(I+E\left(I-E E^{d}\right)\right)^{-1} \varphi(0) & \\
+\int_{0}^{t} Y(t-\theta)\left(I+E\left(I-E E^{d}\right)\right)^{-1} & 0 \leq t \leq \tau,
\end{array}\right.
$$

where $Y(t)$ is the second class of foundation solution of singular differential systems with delay.

Proof. Since $Y(t)$ is the second class of foundation solution of singular differential systems with delay, $Y(t)$ satisfies matrix equations (9). Take Laplace transform for (9), we have

$$
\begin{aligned}
& \int_{0}^{\infty} e^{-\lambda t}(E \dot{Y}(t)) d t \\
&= E \int_{0}^{\infty} e^{-\lambda t} \dot{Y}(t) d t \\
&= E \lambda \int_{0}^{\infty} e^{-\lambda t} Y(t) d t-E Y(0) \\
&= \lambda E L(X(t))-E\left(I-E E^{d}\right), \\
& \int_{0}^{\infty} e^{-\lambda t}\left(A Y(t)+B Y(t-\tau)+\left(I-E E^{d}\right) \delta(t)\right) d t \\
&= A \int_{0}^{\infty} e^{-\lambda t} Y(t) d t \\
&+B \int_{0}^{\infty} e^{-\lambda t} Y(t-\tau) d t+\left(I-E E^{d}\right) \\
&= A L(Y(t)) \\
&+B \int_{-\tau}^{\infty} e^{-\lambda t-\lambda \tau} Y(t) d t+\left(I-E E^{d}\right)
\end{aligned}
$$

$$
\begin{aligned}
= & A L(Y(t)) \\
& +B e^{-\lambda \tau} \int_{0}^{\infty} e^{-\lambda t} Y(t) d t+\left(I-E E^{d}\right) \\
= & \left(A+B e^{-\lambda \tau}\right) L(Y(t))+\left(I-E E^{d}\right) .
\end{aligned}
$$

That is

$$
\begin{aligned}
\lambda E L & (Y(t))-E\left(I-E E^{d}\right) \\
& =\left(A+B e^{-\lambda \tau}\right) L(Y(t))+\left(I-E E^{d}\right) .
\end{aligned}
$$

Because $(E, A)$ is regular, for $\operatorname{real}(\lambda)$ large enough, $\lambda E-A-$ $B e^{-\lambda \tau}$ is invertible, so, we have

$$
L(Y(t))=\left(\lambda E-A-B e^{-\lambda \tau}\right)^{-1}(I+E)\left(I-E E^{d}\right) .
$$

If $x_{2}(t)$ is the solution of (18), we have

$$
\begin{aligned}
\int_{0}^{\infty} e^{-\lambda t}\left(E \dot{x}_{2}(t)\right) d t & =E \int_{0}^{\infty} e^{-\lambda t} \dot{x}_{2}(t) d t \\
& =E \lambda \int_{0}^{\infty} e^{-\lambda t} x_{2}(t) d t-E x_{2}(0) \\
& =\lambda E L\left(x_{2}(t)\right)-E\left(I-E E^{d}\right) \varphi(0),
\end{aligned}
$$$$
\int_{0}^{\infty} e^{-\lambda t}\left(E \dot{x}_{2}(t)\right) d t=\int_{0}^{\tau} e^{-\lambda t}\left(E \dot{x}_{2}(t)\right) d t
$$$$
+\int_{\tau}^{\infty} e^{-\lambda t}\left(E \dot{x}_{2}(t)\right) d t
$$$$
=A \int_{0}^{\tau} e^{-\lambda t} x_{2}(t) d t
$$$$
+\int_{0}^{\tau}\left(I-E E^{d}\right) B e^{-\lambda t} \varphi(t-\tau) d t
$$$$
+A \int_{\tau}^{\infty} e^{-\lambda t} x_{2}(t) d t
$$$$
+B \int_{\tau}^{\infty} e^{-\lambda t} x_{2}(t-\tau) d t
$$$$
=A \int_{0}^{\infty} e^{-\lambda t} x_{2}(t) d t
$$$$
+B e^{-\lambda \tau} \int_{0}^{\infty} e^{-\lambda t} x_{2}(t) d t
$$$$
+\int_{0}^{\tau}\left(I-E E^{d}\right) B e^{-\lambda t} \varphi(t-\tau) d t
$$$$
=A L\left(x_{2}(t)\right)+B e^{-\lambda \tau} L\left(x_{2}(t)\right)
$$$$
+\int_{0}^{\tau}\left(I-E E^{d}\right) B e^{-\lambda t} \varphi(t-\tau) d t .
$$ 
That is,

$$
\begin{aligned}
(\lambda E- & \left.A-B e^{-\lambda \tau}\right) L\left(x_{2}(t)\right) \\
= & \left(I-E E^{d}\right) E \varphi(0) \\
& +\int_{0}^{\tau}\left(I-E E^{d}\right) B e^{-\lambda t} \varphi(t-\tau) d t .
\end{aligned}
$$

Then,

$$
\begin{aligned}
L\left(x_{2}(t)\right)= & \left(\lambda E-A-B e^{-\lambda \tau}\right)^{-1}\left(I-E E^{d}\right) E \varphi(0) \\
+ & \int_{0}^{\tau}\left(\lambda E-A-B e^{-\lambda \tau}\right)^{-1} \\
& \times\left(I-E E^{d}\right) B e^{-\lambda t} \varphi(t-\tau) d t .
\end{aligned}
$$

$$
\begin{aligned}
+\int_{0}^{\tau} & \left(\lambda E-A-B e^{-\lambda \tau}\right)^{-1}(E+I) \\
& \times\left(I-E E^{d}\right)\left(I+E\left(I-E E^{d}\right)\right)^{-1} \\
& \times B e^{-\lambda t} \varphi(t-\tau) d t .
\end{aligned}
$$

from (34) and (38), we know

$$
\begin{gathered}
L\left(x_{2}(t)\right)=L(Y(t))\left(I+E\left(I-E E^{d}\right)\right)^{-1} E \varphi(0) \\
+\int_{0}^{\tau} L(Y(t))\left(I+E\left(I-E E^{d}\right)\right)^{-1} \\
\times B e^{-\lambda \theta} \varphi(\theta-\tau) d \theta .
\end{gathered}
$$

Use auxiliary function $\omega:[-\tau, \infty) \rightarrow[0,1]$ as above, we have

$$
\begin{aligned}
& \int_{0}^{\tau} L(Y(t))\left(I+E\left(I-E E^{d}\right)\right)^{-1} B e^{-\lambda \theta} \varphi(\theta-\tau) d \theta \\
&= L(Y(t)) \\
& \times \int_{0}^{\tau} e^{-\lambda \theta}\left(I+E\left(I-E E^{d}\right)\right)^{-1} B \varphi(\theta-\tau) d \theta \\
&= L(Y(t)) \\
& \times \int_{0}^{\infty} e^{-\lambda \theta}\left(I+E\left(I-E E^{d}\right)\right)^{-1} \\
& \quad \times B \varphi(\theta-\tau) \omega(\theta-\tau) d \theta \\
&= L(Y(t)) L\left(\left(I+E\left(I-E E^{d}\right)\right)^{-1}\right. \\
& \quad\times B \varphi(t-\tau) \omega(t-\tau))
\end{aligned}
$$

$$
\begin{gathered}
=L\left(\int_{0}^{t} Y(t-\theta)\left(I+E\left(I-E E^{d}\right)\right)^{-1}\right. \\
\times B \varphi(\theta-\tau) \omega(\theta-\tau) d \theta) .
\end{gathered}
$$

Then, we have

$$
\begin{aligned}
x_{2}(t)= & Y(t)\left(I+E\left(I-E E^{d}\right)\right)^{-1} E \varphi(0) \\
+ & \int_{0}^{t} Y(t-\theta)\left(I+E\left(I-E E^{d}\right)\right)^{-1} \\
& \times B \varphi(\theta-\tau) \omega(\theta-\tau) d \theta .
\end{aligned}
$$

When $t \geq \tau$,

$$
\begin{aligned}
x_{2}(t)= & Y(t)\left(I+E\left(I-E E^{d}\right)\right)^{-1} E \varphi(0) \\
+ & \int_{-\tau}^{0} Y(t-\theta-\tau)\left(I+E\left(I-E E^{d}\right)\right)^{-1} \\
& \times B \varphi(\theta) d \theta,
\end{aligned}
$$

when $0 \leq t \leq \tau$,

$$
\begin{aligned}
x_{2}(t)= & Y(t)\left(I+E\left(I-E E^{d}\right)\right)^{-1} E \varphi(0) \\
& +\int_{0}^{t} Y(t-\theta)\left(I+E\left(I-E E^{d}\right)\right)^{-1} B \varphi(\theta-\tau) d \theta .
\end{aligned}
$$

This will complete the proof of Lemma 15.

From Lemma 13, 14 and 15 we can easily see that Theorem 12 is true.

Just as the proof of Theorem 12, we can prove that.

Theorem 16. Suppose that matrix couple $(A, E)$ is regular, then the solution of (7) can be written as

$$
\begin{gathered}
\widehat{x}(t)=\int_{0}^{t} X(t-\theta) E^{d}[D u(\theta)+f(\theta)] d \theta \\
+\int_{0}^{t} Y(t-\theta)\left(I+E\left(I-E E^{d}\right)\right)^{-1} \\
\quad \times[D u(\theta)+f(\theta)] d \theta,
\end{gathered}
$$

where $X(t)$ is the first class of foundation solution of singular differential systems with delay and $Y(t)$ is the second class of foundation solution of singular differential systems with delay.

From Lemma 5 and Theorems 12 and 16, we have the following. 
Theorem 17. Suppose that matrix couple $(A, E)$ is regular, then $x(t)$ is the solution of (2), when $t \geq \tau$,

$$
\begin{gathered}
x(t)=\left[X(t)+Y(t)\left(I+E\left(I-E E^{d}\right)\right)^{-1} E\right] \varphi(0) \\
+\int_{-\tau}^{0}\left[X(t-\theta-\tau) E^{d} B+Y(t-\theta-\tau)\right. \\
\left.\quad \times\left(I+E\left(I-E E^{d}\right)\right)^{-1} B\right] \varphi(\theta) d \theta \\
+\int_{0}^{t}\left[X(t-\theta) E^{d}+Y(t-\theta)\right. \\
\left.\quad \times\left(I+E\left(I-E E^{d}\right)\right)^{-1}\right][D u(\theta)+f(\theta)] d \theta,
\end{gathered}
$$

when $0 \leq t \leq \tau$,

$$
\begin{aligned}
x(t)= & {\left[X(t)+Y(t)\left(I+E\left(I-E E^{d}\right)\right)^{-1} E\right] \varphi(0) } \\
& +\int_{0}^{t}\left[X(t-\theta) E^{d} B+Y(t-\theta)\right. \\
& \left.\quad \times\left(I+E\left(I-E E^{d}\right)\right)^{-1} B\right] \varphi(\theta-\tau) d \theta \\
+ & \int_{0}^{t}\left[X(t-\theta) E^{d}+Y(t-\theta)\right. \\
& \left.\quad \times\left(I+E\left(I-E E^{d}\right)\right)^{-1}\right][D u(\theta)+f(\theta)] d \theta,
\end{aligned}
$$

where $X(t)$ is the first class of foundation solution of singular differential systems with delay and $Y(t)$ is the second class of foundation solution of singular differential systems with delay.

Remark 18. As the illustration in Section 2, if $(E, A)$ is regular, by the method of step by step, we can prove that both (8) and (9) are uniquely solvable. We can see that for any consistent initial function $\varphi(t)$, Theorem 17 will be true. Such expression of general solution of (2) will be very useful in the study of the characteristic of (2), such as the observability, the controllability, the optimal control, and so on.

\section{Observability of Singular Differential Systems with Delay}

Now, let us discuss observability of singular differential systems with delay (2) and (3). We work over two cases: $t \geq \tau$ and $0 \leq t<\tau$.

Case $1(t \geq \tau)$. From (3) and Theorem 17, we have

$$
\begin{array}{r}
y(t)=C\left[X(t)+Y(t)\left(I+E\left(I-E E^{d}\right)\right)^{-1} E\right] \varphi(0) \\
+\int_{-\tau}^{0} C\left[X(t-\theta-\tau) E^{d} B+Y(t-\theta-\tau)\right. \\
\left.\times\left(I+E\left(I-E E^{d}\right)\right)^{-1} B\right] \varphi(\theta) d \theta
\end{array}
$$

$$
\begin{gathered}
+\int_{0}^{t} C\left[X(t-\theta) E^{d}+Y(t-\theta)\right. \\
\left.\times\left(I+E\left(I-E E^{d}\right)\right)^{-1}\right] \\
\times[D u(\theta)+f(\theta)] d \theta+H u(t) .
\end{gathered}
$$

That is,

$$
\begin{gathered}
C\left[X(t)+Y(t)\left(I+E\left(I-E E^{d}\right)\right)^{-1} E\right] \varphi(0) \\
+\int_{-\tau}^{0} C\left[X(t-\theta-\tau) E^{d} B+Y(t-\theta-\tau)\right. \\
\left.\times\left(I+E\left(I-E E^{d}\right)\right)^{-1} B\right] \varphi(\theta) d \theta \\
=y(t)-\int_{0}^{t} C\left[X(t-\theta) E^{d}+Y(t-\theta)\right. \\
\left.\times\left(I+E\left(I-E E^{d}\right)\right)^{-1}\right] \\
\times[D u(\theta)+f(\theta)] d \theta-H u(t) .
\end{gathered}
$$

From that, we can see that the problem of system observability can be addressed when $u(\cdot)$ and $f(\cdot)$ are identically zero. Thus, we can assume, with no loss of generality, that $u(\cdot) \equiv 0, f(\cdot) \equiv 0$ and study the observability of systems (6) and

$$
y(t)=C x(t)
$$

Then, we have

$$
\begin{array}{r}
y(t)=C\left[X(t)+Y(t)\left(I+E\left(I-E E^{d}\right)\right)^{-1} E\right] \varphi(0) \\
+\int_{-\tau}^{0} C\left[X(t-\theta-\tau) E^{d} B+Y(t-\theta-\tau)\right. \\
\left.\times\left(I+E\left(I-E E^{d}\right)\right)^{-1} B\right] \varphi(\theta) d \theta .
\end{array}
$$

From Lemma 8, we have

$$
\begin{aligned}
& \int_{0}^{l} C[X(t-\theta+\tau)+Y(t-\theta+\tau) \\
& \left.\times\left(I+E\left(I-E E^{d}\right)\right)^{-1} E\right] \varphi(\theta-\tau) \delta(l-\theta) d \theta \\
& =C[X(t-l+\tau)+Y(t-l+\tau) \\
& \left.\quad \times\left(I+E\left(I-E E^{d}\right)\right)^{-1} E\right] \varphi(l-\tau) .
\end{aligned}
$$

Let $l=\tau$; then

$$
\begin{aligned}
& \int_{0}^{\tau} C[X(t-\theta+\tau)+Y(t-\theta+\tau) \\
&\left.\times\left(I+E\left(I-E E^{d}\right)\right)^{-1} E\right] \varphi(\theta-\tau) \delta(\tau-\theta) d \theta \\
&= C\left[X(t)+Y(t)\left(I+E\left(I-E E^{d}\right)\right)^{-1} E\right] \varphi(0),
\end{aligned}
$$


that is,

$$
\begin{aligned}
C[X(t)+Y(t) & \left.\left(I+E\left(I-E E^{d}\right)\right)^{-1} E\right] \varphi(0) \\
=\int_{-\tau}^{0} C[X(t-\theta)+Y(t-\theta) & \left.\times\left(I+E\left(I-E E^{d}\right)\right)^{-1} E\right] \delta(-\theta) \varphi(\theta) d \theta .
\end{aligned}
$$

Taking it into (50), we have

$$
\begin{aligned}
y(t)=\int_{-\tau}^{0} C[X(t-\theta)+Y(t-\theta) & \left.\times\left(I+E\left(I-E E^{d}\right)\right)^{-1} E\right] \delta(-\theta) \varphi(\theta) d \theta \\
+\int_{-\tau}^{0} C\left[X(t-\theta-\tau) E^{d} B+Y(t-\theta-\tau)\right. & \left.\times\left(I+E\left(I-E E^{d}\right)\right)^{-1} B\right] \varphi(\theta) d \theta \\
=\int_{-\tau}^{0} C[ & X(t-\theta)+Y(t-\theta) \\
& \left.\times\left(I+E\left(I-E E^{d}\right)\right)^{-1} E\right] \delta(-\theta) \\
& +X(t-\theta-\tau) E^{d} B+Y(t-\theta-\tau) \\
& \left.\times\left(I+E\left(I-E E^{d}\right)\right)^{-1} B\right] \varphi(\theta) d \theta .
\end{aligned}
$$

Let

$$
\begin{aligned}
& W(t, \theta)=[X(t-\theta)+Y(t-\theta) \\
&\left.\times\left(I+E\left(I-E E^{d}\right)\right)^{-1} E\right] \delta(-\theta) \\
&+X(t-\theta-\tau) E^{d} B+Y(t-\theta-\tau) \\
& \times\left(I+E\left(I-E E^{d}\right)\right)^{-1} B
\end{aligned}
$$

Then,

$$
y(t)=\int_{-\tau}^{0} C W(t, \theta) \varphi(\theta) d \theta
$$

So, we have

$$
\begin{aligned}
\int_{-\tau}^{0} W^{T}(t, s) C^{T} y(t) d s \\
\quad=\int_{-\tau}^{0}\left[\int_{-\tau}^{0} W^{T}(t, s) C^{T} C W(t, \theta) \varphi(\theta) d \theta\right] d s \\
\quad=\int_{-\tau}^{0}\left[\int_{-\tau}^{0} W^{T}(t, s) C^{T} C W(t, \theta) \varphi(\theta) d s\right] d \theta
\end{aligned}
$$

$$
\begin{aligned}
& =\int_{-\tau}^{0}\left[\int_{-\tau}^{0} W^{T}(t, \theta) C^{T} C W(t, s) \varphi(s) d \theta\right] d s \\
& =\int_{-\tau}^{0}\left[\int_{-\tau}^{0} W^{T}(t, \theta) C^{T} C W(t, s) d \theta\right] \varphi(s) d s .
\end{aligned}
$$

That is,

$$
\begin{aligned}
& \int_{-\tau}^{0}\left[\int_{\tau}^{t_{1}} W^{T}(t, s) C^{T} y(t) d t\right] d s \\
& \quad=\int_{-\tau}^{0}\left[\int_{\tau}^{t_{1}}\left[\int_{-\tau}^{0} W^{T}(t, \theta) C^{T} C W(t, s) d \theta\right] d t\right] \varphi(s) d s
\end{aligned}
$$

Let

$$
Q\left(t_{1}, s\right)=\int_{\tau}^{t_{1}}\left[\int_{-\tau}^{0} W^{T}(t, \theta) C^{T} C W(t, s) d \theta\right] d t .
$$

Then,

$$
\begin{gathered}
\int_{-\tau}^{0}\left[\int_{\tau}^{t_{1}} W^{T}(t, s) C^{T} y(t) d t\right] d s \\
=\int_{-\tau}^{0} Q\left(t_{1}, s\right) \varphi(s) d s .
\end{gathered}
$$

From that, we have the main result of this paper as follows.

Theorem 19. Singular differential system with delay (6) and (49) is observable in $\left[\tau, t_{1}\right]$ if the observability matrix

$$
Q\left(t_{1}, s\right)=\int_{\tau}^{t_{1}}\left[\int_{-\tau}^{0} W^{T}(t, \theta) C^{T} C W(t, s) d \theta\right] d t
$$

has rank $n$ for all $s \in[-\tau, 0]$. Where

$$
\begin{aligned}
& W(t, \theta)=[X(t-\theta)+Y(t-\theta) \\
&\left.\times\left(I+E\left(I-E E^{d}\right)\right)^{-1} E\right] \delta(-\theta) \\
&+X(t-\theta-\tau) E^{d} B \\
&+Y(t-\theta-\tau)\left(I+E\left(I-E E^{d}\right)\right)^{-1} B
\end{aligned}
$$

where $X(t)$ is the first class of foundation solution of singular differential system with delay and $Y(t)$ is the second class of foundation solution of singular differential system with delay.

Proof. If the observability matrix

$$
Q\left(t_{1}, s\right)=\int_{\tau}^{t_{1}}\left[\int_{-\tau}^{0} W^{T}(t, \theta) C^{T} C W(t, s) d \theta\right] d t
$$

has rank $n$ for all $s \in[-\tau, 0]$, then $Q^{-1}\left(t_{1}, s\right)$ exists, and from (60), we can take

$$
\varphi(s)=Q^{-1}\left(t_{1}, s\right) \int_{\tau}^{t_{1}} W^{T}(t, s) C^{T} y(t) d t,
$$

the singular differential system with delay (6) and (49) is observable in $\left[\tau, t_{1}\right]$. 
Theorem 20. If there exist $s_{1}, s_{2} \in[-\tau, 0]$, and $s_{1}<s_{2}$ such that when $s \in\left(s_{1}, s_{2}\right)$, the observability matrix $Q\left(t_{1}, s\right)$, $\operatorname{rank} Q\left(t_{1}, s\right)<n$, then singular differential system with delay (6) and (49) is unobservable.

Proof. For $\operatorname{rank} Q\left(t_{1}, s\right)<n\left(s \in\left(s_{1}, s_{2}\right)\right)$, there exists $\alpha(s) \in$ $R^{n}, \alpha(s) \neq 0$, such that $Q\left(t_{1}, s\right) \alpha(s)=0$.

Let $\varphi_{1}(s)$ satisfy $(60)$ for any $y(t)$, and

$$
\varphi_{2}(s)= \begin{cases}\varphi_{1}(s), & s \notin\left(s_{1}, s_{2}\right), \\ \varphi_{1}(s)+\alpha(s), & s \in\left(s_{1}, s_{2}\right) .\end{cases}
$$

Obviously, for $s \in[-\tau, 0], \varphi_{1}(s) \neq \varphi_{2}(s)$, and

$$
\begin{aligned}
\int_{-\tau}^{0} Q & \left(t_{1}, s\right) \varphi_{2}(s) d s \\
& =\int_{-\tau}^{0} Q\left(t_{1}, s\right) \varphi_{1}(s) d s+\int_{s_{1}}^{s_{2}} Q\left(t_{1}, s\right) \alpha(s) d s \\
& =\int_{-\tau}^{0} Q\left(t_{1}, s\right) \varphi_{1}(s) d s \\
& =\int_{-\tau}^{0}\left[\int_{\tau}^{t_{1}} W^{T}(t, s) C^{T} y(t) d t\right] d s .
\end{aligned}
$$

That is for any $y(t)$, we can have more than one $\varphi(s)$ in (60), so singular differential system with delay (6) and (49) is unobservable.

Case $2(0 \leq t<\tau)$. From (3) and Theorem 17, we have

$$
\begin{gathered}
y(t)=C\left[X(t)+Y(t)\left(I+E\left(I-E E^{d}\right)\right)^{-1} E\right] \varphi(0) \\
+\int_{-\tau}^{t-\tau} C\left[X(t-\theta-\tau) E^{d} B\right. \\
\left.+Y(t-\theta-\tau)\left(I+E\left(I-E E^{d}\right)\right)^{-1} B\right] \\
\quad \times \varphi(\theta) d \theta \\
+\int_{0}^{t} C\left[X(t-\theta) E^{d}\right. \\
\left.\quad+Y(t-\theta)\left(I+E\left(I-E E^{d}\right)\right)^{-1}\right] \\
\times[D u(\theta)+f(\theta)] d \theta+H u(t) .
\end{gathered}
$$

That is,

$$
\begin{aligned}
C\left[X(t)+Y(t)\left(I+E\left(I-E E^{d}\right)\right)^{-1} E\right] \varphi(0) \\
+\int_{-\tau}^{t-\tau} C\left[X(t-\theta-\tau) E^{d} B+Y(t-\theta-\tau)\right. \\
\left.\times\left(I+E\left(I-E E^{d}\right)\right)^{-1} B\right] \varphi(\theta) d \theta
\end{aligned}
$$

$$
\begin{gathered}
=y(t)-\int_{0}^{t} C\left[X(t-\theta) E^{d}+Y(t-\theta)\right. \\
\left.\times\left(I+E\left(I-E E^{d}\right)\right)^{-1}\right] \\
\times[D u(\theta)+f(\theta)] d \theta-H u(t) .
\end{gathered}
$$

From (68), we can easily find that when $\theta \in(t-\tau, 0)$, we cannot get any information about $\varphi(\theta)$. That is, the state initial function $\varphi(t)$ in $[-\tau, 0]$ cannot be uniquely determined from the knowledge of the control $u(\cdot)$ and observation $y(\cdot)$ over $[0, \tau)$, singular differential control systems with delay (2) and (3) are not observable on $[0, \tau)$.

\section{Conclusion}

In this paper, we study linear singular differential systems with delay in the state equations. We give a general description of the solutions and as the use of such result, we derive a necessary and sufficient condition for exact observability in finite time.

In [7], the problem, finding general solutions of singular systems with delay, was presented for the first time. But the result and the approach of our paper are brand-new, which may have significant advantages in comparison with the results of [7]. For example, it can be easily used to study the observability of singular control systems with delay.

For the observability of singular control systems with delay, we study it in two cases. When $t \geq \tau$, we give a necessary and sufficient condition. When $0 \leq t<\tau$, we point out that singular differential control systems with delay (2) and (3) are not observable. These will be very useful in the study of singular control systems with delay, and that could also be regarded as the example of the use of the general description of the solutions of singular differential systems with delay.

\section{Acknowledgments}

This research has been supported by the National Natural Science Foundation of China (no. 11371027 and no. 11071001), Doctoral Fund of the Ministry of Education of China (no. 20093401110001), and the Major Program of Educational Commission of Anhui Province of China (no. KJ2010ZD02).

\section{References}

[1] J. K. Hale and S. M. V. Lunel, "Strong stabilization of neutral functional differential equations," IMA Journal of Mathematical Control and Information, vol. 19, no. 1-2, pp. 5-23, 2002.

[2] Z. Zuxiu, Theory of Functional Differential Equations, AnHui Education Press, Hefei, China, 1994.

[3] J. Wei, The Degenerate Differential Systems with Delay, University of Anhui Press, Hefei, China, 1998.

[4] M. Malek-Zavarei and M. Jamshidi, Time-Delay Systems: Analysis, Optimization and Application, vol. 9 of North-Holland Systems and Control Series, North-Holland, Amsterdam, The Netherlands, 1987. 
[5] V. Răsvan, "Time-delay systems with remarkable structural properties," IMA Journal of Mathematical Control and Information, vol. 29, no. 2, pp. 271-289, 2012.

[6] L. Dai, Singular Control Systems, vol. 118 of Lecture Notes in Control and Information Sciences, Springer, Berlin, Germany, 1989.

[7] S. L. Campbell, Singular Systems of Differential Equations. II, vol. 61 of Research Notes in Mathematics, Pitman, Boston, Mass, USA, 1982.

[8] C.-J. Wang and H.-E. Liao, "Impulse observability and impulse controllability of linear time-varying singular systems," Automatica, vol. 37, no. 11, pp. 1867-1872, 2001.

[9] W. Wang and Y. Zou, "Analysis of impulsive modes and Luenberger observers for descriptor systems," Systems \& Control Letters, vol. 44, no. 5, pp. 347-353, 2001.

[10] J. Wei and S. Wenzhong, "Controllability of singular systems with control delay," Automatica, vol. 37, no. 11, pp. 1873-1877, 2001.

[11] J. Wei, "Eigenvalue and stability of singular differential delay systems," Journal of Mathematical Analysis and Applications, vol. 297, no. 1, pp. 305-316, 2004.

[12] J. Wei, "A variation formula for time varying singular delay differential systems," Chinese Annals of Mathematics. Series A, vol. 24, no. 2, pp. 161-166, 2003.

[13] J. Wei and Z. X. Zheng, "The general solution for the degenerate differential system with delay," Acta Mathematica Sinica, vol. 42, no. 5, pp. 769-780, 1999.

[14] J. Wei and Z. X. Zheng, "The variation-of-constants formula and the general solution of degenerate neutral differential systems," Acta Mathematicae Applicatae Sinica, vol. 21, no. 4, pp. 562-570, 1998.

[15] J. Wei and Z. Zheng, "On the degenerate differential system with delay," Annals of Differential Equations, vol. 14, no. 2, pp. 204211, 1998.

[16] J. Wei and Z. C. Wang, "Controllability of singular control systems with delay," Journal of Hunan University. Natural Sciences, vol. 26, no. 4, pp. 6-9, 1999.

[17] J. Wei, "Function-controllability of nonlinear singular delay differential control systems," Acta Mathematica Sinica, vol. 49, no. 5, pp. 1153-1162, 2006.

[18] J. Wei, "On the solvability of singular differential delay systems with variable coefficients," International Journal of Dynamical Systems and Differential Equations, vol. 1, no. 4, pp. 245-249, 2008.

[19] J. Wei, "The constant variation formulae for singular fractional differential systems with delay," Computers \& Mathematics with Applications, vol. 59, no. 3, pp. 1184-1190, 2010. 


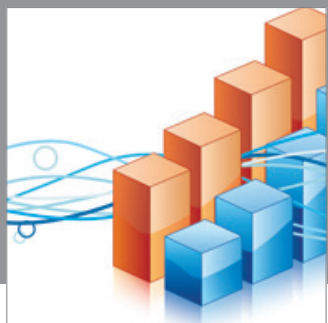

Advances in

Operations Research

mansans

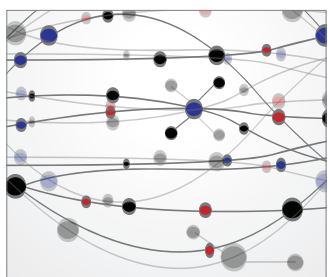

The Scientific World Journal
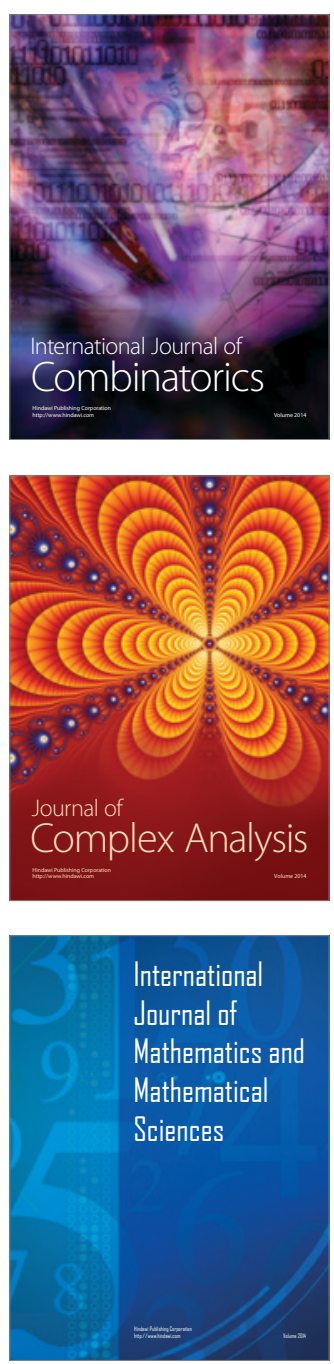
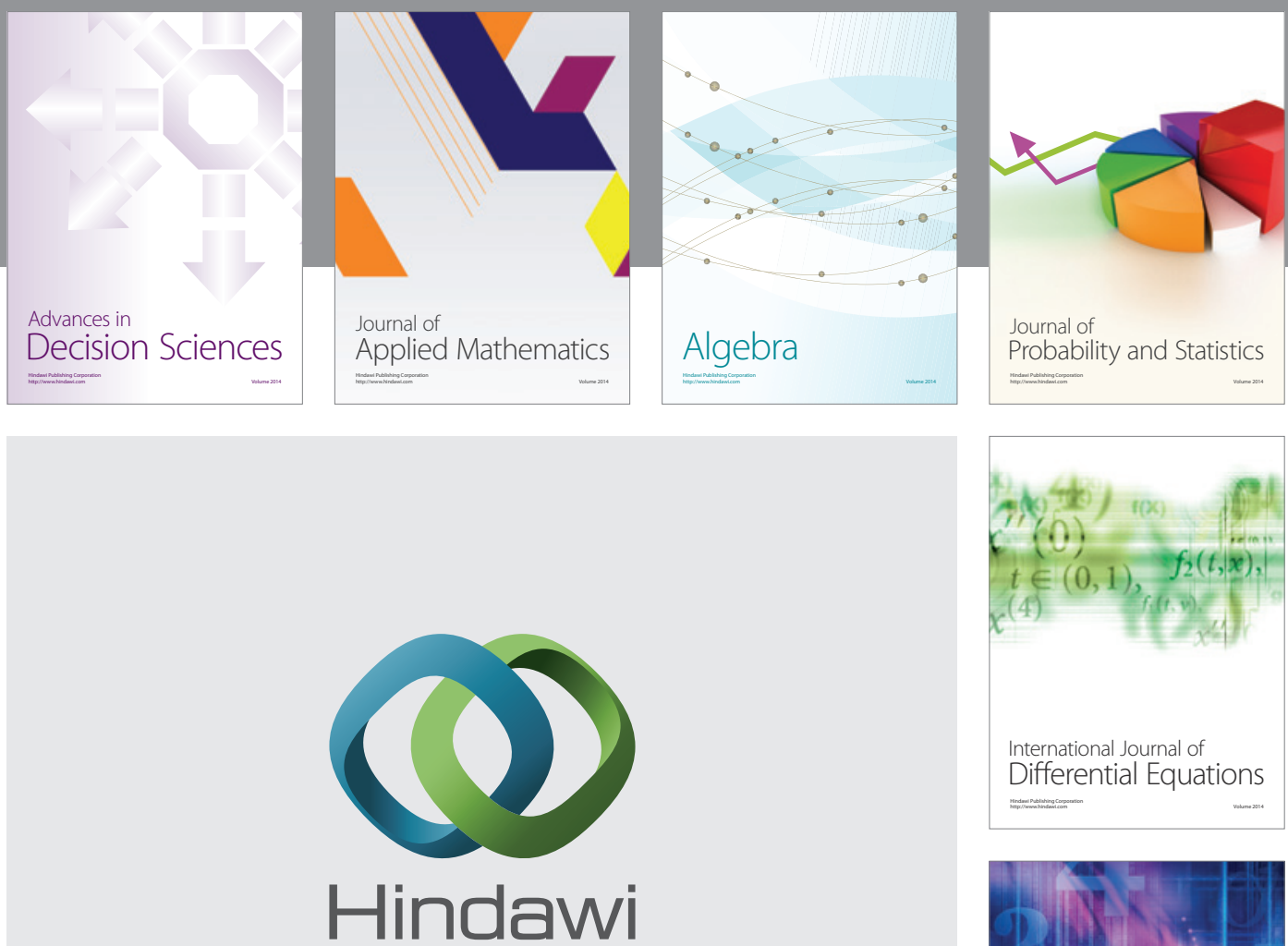

Submit your manuscripts at http://www.hindawi.com
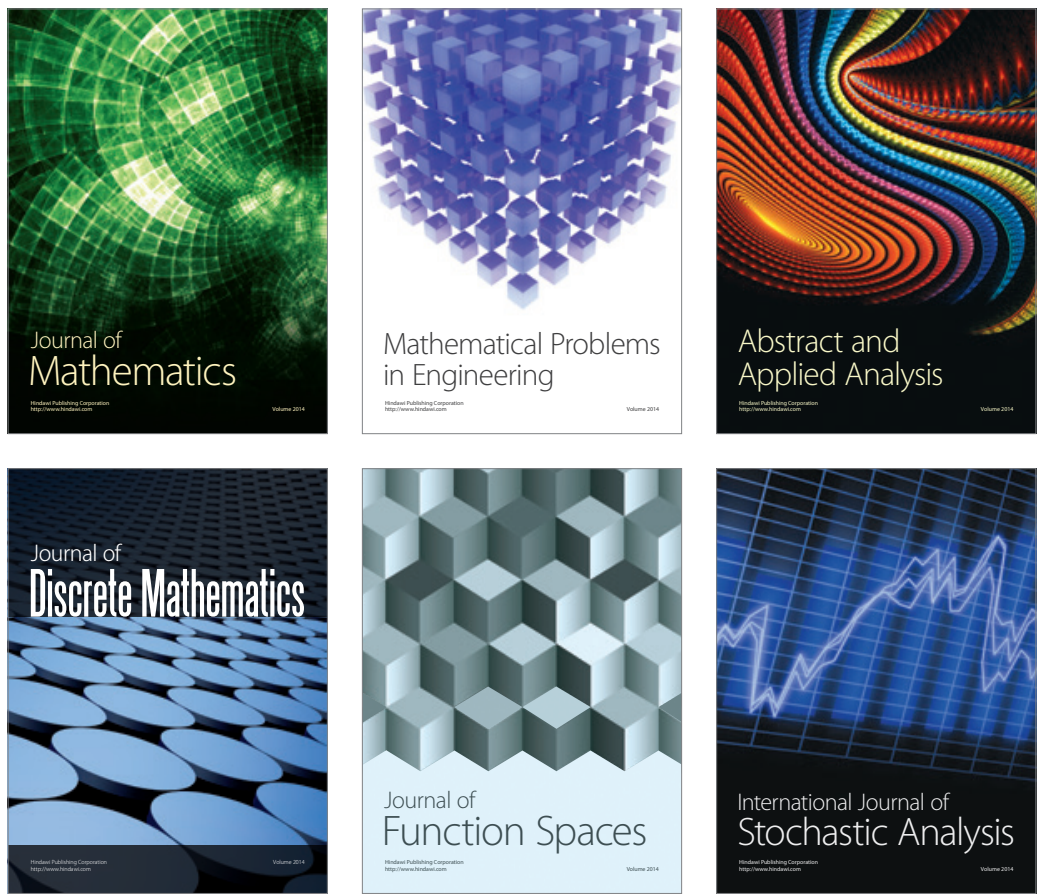

Journal of

Function Spaces

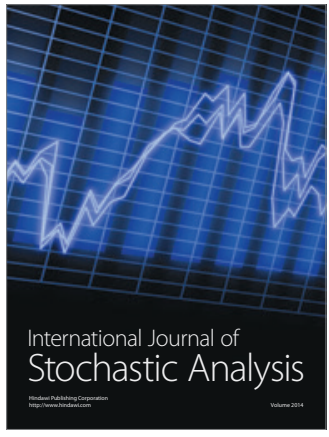

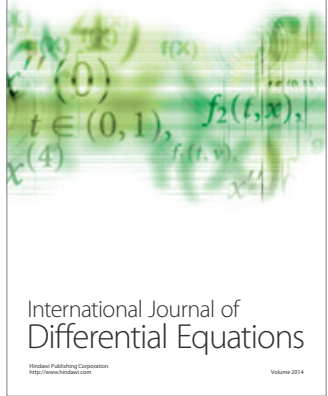
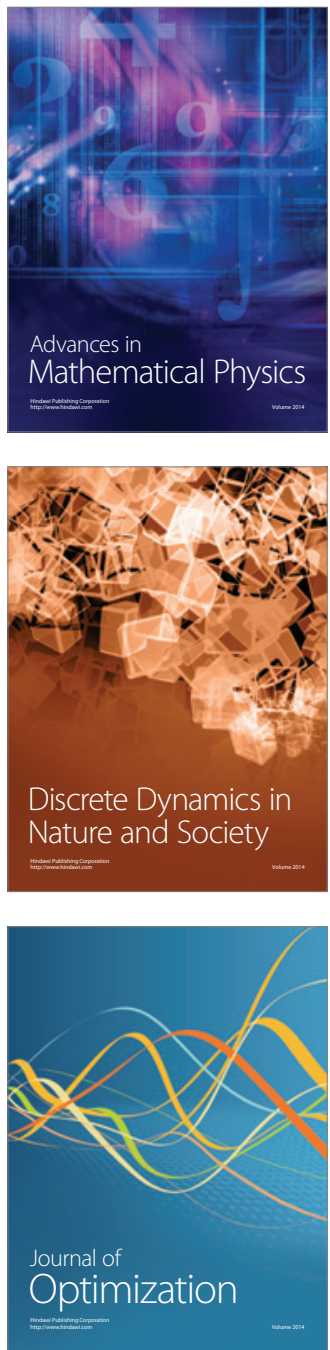